\title{
Improving Factors Affecting the Development of Corporate Entrepreneurship in the Housing Sector Using QFD
}

\author{
Abbas Matloubi ${ }^{1}$ \\ ${ }^{1}$ Department of East Studies, Azerbaijan National Academy of Sciences University, Baku, Azerbaijan \\ Correspondence: Abbas Matloubi, Department of East Studies, Azerbaijan National Academy of Sciences \\ University, Baku, Azerbaijan. E-mail: Abbas.Matloubi@gmail.com
}

\author{
Received: April 20, $2015 \quad$ Accepted: April 22, $2015 \quad$ Online Published: August 30, 2015 \\ doi:10.5539/mas.v9n9p220 URL: http://dx.doi.org/10.5539/mas.v9n9p220
}

\begin{abstract}
A new dimension of entrepreneurial activities in the business has been considered in business since the early $80 \mathrm{~s}$, is known as the Corporate Entrepreneurship (CE). This type of entrepreneurship is very similar to the independent entrepreneur (in the form of running a new business), and the only difference is that the entrepreneur acts within an organization that already worked. Corporate entrepreneurship is a process through which organizations are realizing the opportunities and manage production factors to create additional value in innovative ways. Housing sector in Iran, with abundant employment opportunities, can contribute much role in the current situation, but lack of attention to the concept of entrepreneurship in the field of employment in this sector has caused the traditional way of thinking dominate this sector. This research has been trying to use the efficient management models such as quality function deployment model offer strategies for promotion of corporate entrepreneurship in housing sector. The study population consists of all directors and employees of two Housing Investment Companies in Tehran. A number of 137 questionnaires were collected after applying the sampling formula to sampling, and the results showed that the dimensions of innovation, being active, management structure and philosophy of bounces are in very good conditions but the dimensions of risking, strategic orientation, Resource-based orientation and entrepreneurial culture require improvement. A number of 14 recovery strategies for the promotion of the 4 dimensions were designed using 11 managers of the both under study companies. Strategies appropriate measure of relative weight and absolute weight are appropriate criteria for ranking, among the strategies the three strategies including (accurate monitoring of the implementation of the law), (creating a culture of collaboration and knowledge sharing between employees) and outsourcing CE consultancy to professional organizations) have the highest weight and influence, and more input parameters will be recovered by their implementation.
\end{abstract}

Keywords: corporate entrepreneurship, housing sector, quality function deployment model

\section{Introduction}

Today, unlike the past, global markets are faced with daily or even in moments changes of human community's needs, and manufactures and service providers that meet these needs should move forward quickly with brilliant goal setting to keep pace with the rapid changes and survive and grow in these markets, since the economic structure of the world today is radically different from that of the past (Farhangi \& Safarzadeh, 2005). Hardware indexes and tangible assets that in past were determining factors in the economic position of a firm have replaced with innovation, invention, new products generation and software asset (fox, 2008). If yesterday the richest people in the world were those who had more financial resources, today, the richest people in the world are those knowledge-oriented and entrepreneurs. Thus, today's Economic Development is based on innovation and knowledge. Meanwhile, organizations survival and life requires new approaches and solutions and entrepreneurship as a strategic approach and an advantage in economies systems can be considered as an efficient model to achieve social and economic development objectives (Peltola, 2012). Entrepreneurship is as an engine of economic development momentum, since it is necessary to all corporations. The reason could be the more competitive world of the day that makes corporations to keep pace with the advancements making use of entrepreneurs. The world experience has shown that important innovations and technological advances will not happen in large government organizations and corporations because the ability to adapt to the market demand is not obtained in large, complex and nested structures. As a result, the only effective way is to develop conditions 
that small economic activities can be established and compete to obtain the rapid development and delivery of new products (Rahmati, 2011). Also in today's world, factors affecting the development of entrepreneurship in society and the unique characteristics of entrepreneurs in the organization are considered as an element of different aspects of economic, social, cultural, and political development; so that, in one hand, corporate entrepreneurship is seen as the production and corporate development motor element and on the other hand, nurturing and strengthening the investment morale and productivity in human capital (Ghahramani et al., 2010).

$\mathrm{CE}$ is process through which organizations are realizing the opportunities and manage the production agents to create additional value in creative ways. Those corporations with innovation, risking-taking, leading, fearlessness and aggressively have the entrepreneurial capability and in support of the above conditions attach great importance to technological leadership, research and development, that all stems from the fact that these organizations should apply to people with more knowledge than peers (Lumpkin \& Lichtenstein, 2005).

The housing market in metropolitan cities has faced with significant changes in recent years and often changes in housing prices after a period of 3 to 4 years, with inflation, rises in a staircase way. Given the large share of the housing cost of urban household incomes, especially in metropolitans (over $50 \%$ of total costs) households are faced with many problems in the stepwise increase in housing prices (which increase the rent will follow). On the other hand, about 110 activities have been identified in relation to housing sector and any downturn in the market results in unemployment of a large number of persons employed in activities related to this sector. Therefore, the government has sought to present strategies to promote $\mathrm{CE}$ and as a result the development of the housing sector employment. Consequently, this study will attempt to provide appropriate strategies in this regard using the quality function deployment (QFD) and in-depth interviews in the model.

\section{Literature Review and Concepts}

Fox, (2008) investigated the relationship between CE and performance to develop the financial and non-financial performance indexes and thus the satisfaction of stakeholders by entrepreneurship promotion.In his study, he mentions that due to the multidimensional nature of organizations stakeholder, $\mathrm{CE}$ cannot be measured in one simplistic dimension, so his article offers $8 \mathrm{CE}$ dimensions that are used in this research, as well. They include: innovation, entrepreneurial culture, bounces philosophy, management structure, and resource- based orientation, strategic orientation, risking and being active.

Pang et al (2008) explained the role of government in technological EC. According to them, the firms to gain advantage of emerging business opportunities and maintain it should adopt flexible and innovative strategies to cope with the competitive and the uncertain environment and changing customer demands as well. However, the performance is not as expected. Many firms that have used technological EC strategies had failed. They criticized Antonin and Prod a definition technological EC: "Some researchers believe that entrepreneurship does not necessarily involve the organization or firm establishment. In fact, technological EC has become a significant strategy for enterprises to maintain a competitive advantage in today's highly competitive environment. This can be due to the shortening of product life cycles and greater diversity of clients tastes than past. Technological CE from the perspective of exploring opportunities can be defined as "within the organization policy and practice in order to explore and exploit market opportunities for technologies".

Also Montoro-Sánchez \& Soriano (2011) expressed the most important effects of CE in the community as follows:

- Improving the quality of life, exploitation of the benefits and their activation for national productivity

Entrepreneurs continuously innovate and develop products and services needed by people and the efficient use of financial resources for investment in people, while improving quality, paving the way for the promotion of the national productivity.

- The elimination of damages, gaps and bottlenecks in the market and society.

Entrepreneurs with use of creative resources, risking and ambiguity tolerance are able to change threats to opportunities subtlety and discover and exploited new opportunities in difficult conditions such as lack of exchange, inflation, war and etc.

- The balance in a dynamic economy and encourage investment

- The transformation and revitalization of the national and local life

- The reduction of administrative bureaucracy (reducing desk works and encouraging pragmatism, etc.)

- Innovation and change facilitation. 
- The creation of wealth and income distribution

- Employment: when CE starts new jobs to when moved, at least they need one or more workforce to manage their own affairs.

Also Beigi Niya et al (2010) conducted a research with the aim of "the impact of the need on the staff willingness to $\mathrm{CE}$ in the central headquarters of the National Iranian Oil Company". Variables include a variety of needs (need for success / achievement, power, respect, self-actualization, life, and social security) as the independent variables, and the staff willingness to $\mathrm{CE}$ as the dependent variable, respectively. Data being collected, two questioners with tested reliability and validity were used. After correcting and adjusting some of the questions and test-retest reliability and validity of them, they were used in order to assess the need and desire of employees to CE. Cornbrash's alpha coefficient for both questionnaires was $85.65 \%$ and $86.68 \%$, respectively. Using the method of correlation, data analysis software SPSS, and using multiple regression, hypothesis testing with a confidence level of 0.95 was obtained. According to multiple regression method, seven research hypotheses and thus influence of various needs on the willingness of staff to CE was confirmed.

Rahmati (2011) in other research aimed at propose a model for explaining the role of structural, behavioral and contextual (environmental) factors in the process of promoting entrepreneurship in the organization of Islamic Azad University, Science and Research branch for promoting indigenous entrepreneurship model. This study is a descriptive- correlation using a survey with the survey data collected through questionnaires. Descriptive and inferential statistical methods were used in data analysis. The findings show that the Islamic Azad University, Science and Research Branch, Tehran does not have a desirable CE.Correlation relationships confirmed relationships between structural, behavioral (content) and contextual factors and entrepreneurship. The hypothesis test showed that among the factors structural and contextual factors had the maximum and minimum impact on the promotion of entrepreneurship in universities, respectively. The results show that the structure of the Islamic Azad University, Science and Research Branch, Tehran is a formal structure of hierarchy of laws and administrative regulations that is far from necessary conditions for the promotion of corporate entrepreneurship. Thus, the traditional model design presented in this study can help to enhance corporate entrepreneurship based on three structural, behavioral and environmental factors.

Reza Zadeh (2003) in a study examined the relationship between corporate structure and corporate entrepreneurship in The Social Security Organization of Tehran, and obtained the following results:

There was a significant relationship between the complexity of the organizational structure and CE, but the correlation coefficient obtained by Buenaventura curve indicates an inverse relationship between the complexities of the organizational structure and CE. This means that the separation of internal organizational units or categories, the number of management levels, and organizational geographic dispersion has an inverse relationship with the $\mathrm{CE}$. Moreover, there was a significant relationship between formal organizational structure and $\mathrm{CE}$, but the correlation coefficient obtained by Buenaventura curve indicates an inverse relationship between the complexity of the organizational structure and CE.

\section{Results}

According to the literature review, one of the most comprehensive inventories of CE is in the article (Fox, 2008) with a total number of 8 major dimensions have been referenced in numerous articles. Thus, the model according to the following table is used as the main research inventory.

Table 1. Corporate Entrepreneurship Indexes and Dimension (Fax-2008)

Dimension Components

$\begin{array}{lll} & 1-\quad \text { This corporation constantly supplies market with the new products and services. } \\ & 2-\quad \text { Always dramatic changes created in the products and services. } \\ & 3-\quad \text { Typically, the corporation tries to have fit bilateral relations with other organizations. } \\ & 4-\quad \text { The organization compared to other organizations provides market sooner with products, services and } \\ & 5-\quad \text { The senior management of the organization tends to have high-risk projects that are arguably more }\end{array}$


efficient.

6- The senior management of the organization believes that appropriate and prompt response to environmental needs is crucial to achieve organizational goals.

7- When an organization is in a position that must decide in ambiguity, all employees are trying to create a situation that is likely to make the maximum possible use of the opportunities.

8- The organization strategies are based on perception of environmental opportunities and constraints of the organization's resources cannot modify it.

9- Our main task in the organization is to follow perceived opportunities in the environment and allocate sufficient resources to take advantage of it.

10- Environmental opportunities that will determine the organization's business strategies.

11- Since the aim of this organization is the efficient use of resources, often we will act quickly to invest in very large resources.

12- We prefer to use the resources that we have full control and ownership.

13- In the use of opportunities, having more financial resources is more important than having just a raw idea.

14- In this organization, informal control and supervision is a priority.

15- It is important for us to do business activities, even if we ignore the official procedures.

16- We are trying to get along with changes even if it leads to forgetfulness of the organizations' past.

17- Operational management style allows organization to act in a range of formal and informal activities and relations.

18- There is a strong tendency in the organizations to allow employees to conduct their favorite behavior in different situations.

19- The corporate staff is evaluated and reward on the added value created by them.

20- The organization is trying to reward employees so that to benefit from the added value of the organization.

21- There are many innovative ideas for better use of resources recommended by staff in the organization.

22- Goods and services provided by the organization are designed based on adequate information of customers, markets and changes in the community.

23- We never experienced lack of innovative ideas in the organization.

24- The agency staff understands the ways they use to understand the market should always be updated.

25- The agency staff believes that organizational capability for continuous learning can improve performance to an appropriate level.

Since this research have been carried out in real organizations, objective and living (dynamic) cooperation's, and the results can be used in practice, thus it is an applied research and it can be said a descriptive/analytical research of the survey. The method employed in this study is an analytical survey. A combination of library and field research methods was used in order to collect information. Library research is mainly used to study literature and examine familiarity with the history and experiences of entrepreneurial research. At this stage, according to the literature, factors related to CE have been extracted and surveys conducted on the organizations staff, and the status quo per dimensions was extracted; thus, using factor analysis, reliability indicators approved. 
In this study, the dimensions with low performance status were determined and are considered as the input for Quality box and following the steps of the box, improvement strategy per each of them was extracted. It should be noted what QFD introduced as a bargaining chip for many organizations today is nothing but a careful examination of organizations needs from different perspectives, as far as some international organizations are built their reputation based on accurate and efficient use of these tools. QFD is trying to add value to the organization by focusing on the clear understanding of the organization needs and developing of the demands in all phases of the development process from the earliest development of a concept or a problem in the mind of the organization designers.

The population consisted of all employees and directors of the two large house investment companies in Tehran. Given the limited volume of approximately 650 subjects, this study uses the finite population sampling formula to determine the sample size. A preliminary sampling was used to determine the standard deviation of the population. For this purpose, a questionnaire survey was randomly distributed among 42 employees of the target population. The standard deviation of the results of the questionnaire showed that most of the population standard deviation is equal to 0.358 .

$$
n=\frac{N \times Z_{\alpha / 2}^{2} \times \delta^{2}}{\varepsilon^{2}(N-1)+Z_{\alpha / 2}^{2} \times \delta^{2}} \quad \Rightarrow \quad n=\frac{650 \times(1.96)^{2} \times 0.358^{2}}{0.05^{2}(649)+(1.96)^{2} \times 0.358^{2}}=151
$$

Thus, according to the obtained standard deviation, the maximum sample error of 5\%, which is equivalent to 151 , was obtained that from 160 distributed questionnaires a number of 137 completed send collected, and thus the 137 questionnaires were used on statistical analysis.

\section{Discussion}

In this study, Cornbrash's alpha test was used to measure the reliability of the survey questionnaire. Cornbrash's alpha for the CE questionnaire results are presented in the table below:

Table 2. Cornbrash's alpha test (the reliability of the questionnaire) for CE questionnaire

\begin{tabular}{|c|c|c|c|c|}
\hline \multicolumn{4}{|l|}{ Case Processing Summary } & \\
\hline & & $\mathrm{N}$ & $\%$ & \\
\hline Cases & Valid & 137 & 100.0 & \\
\hline & Excluded (a) & 0 & 0 & \\
\hline & Total & 137 & 100.0 & \\
\hline \multicolumn{4}{|c|}{ a List wise deletion based on all variables in the procedure. } & \\
\hline \multicolumn{4}{|c|}{ Reliability Statistics } & \\
\hline \multicolumn{2}{|c|}{ Cornbrash's Alpha } & \multicolumn{2}{|c|}{$\mathrm{N}$ of Items } & $\frac{n}{w}$ \\
\hline \multicolumn{2}{|l|}{.863} & \multicolumn{2}{|c|}{25} & \\
\hline
\end{tabular}

Factor analysis was used to assess the validity of questionnaire. The first test of the factor analysis is Bartlett test, or adequacy of the sampling that assess distribution of questionnaire accuracy. Final results of the factor analysis are as follows:

Table 3. KMO and Bartlett's test of the questionnaire indexes

\begin{tabular}{ll}
\hline 0.709 & dequacy of sampling (KMO) \\
\hline 0.000 & ignificance level \\
\hline
\end{tabular}

After factor analysis, no indication was removed from the final questionnaire and the final questionnaire consisted of 25 questions that were approved. 
Before carrying out any statistical analysis, it is essential to ensure normal or non-normal distribution of data. To do this we must use the Kolmogorov-Smirnov test.According to the table below and in accordance with the significance level that is higher than 0.05 , CE Inventory data is normally distributed, in the following parametric tests should be used for statistical analysis.

Table 4. Kolmogorov-Smirnov test for CE questionnaire One-Sample Kolmogorov-Smirnov Test

\begin{tabular}{ccc}
\hline & & Importance Data \\
\hline \multirow{2}{*}{ Normal Parameters } & $\mathrm{N}$ & 137 \\
& Mean & 5.1719 \\
Most Extreme Differences & Std. Deviation & 1.33492 \\
& Absolute & .224 \\
& Positive & .117 \\
& Negative & -.224 \\
& Kolmogorov-Smirnov Z & 1.268 \\
\hline
\end{tabular}

In this section, the results of data analysis for each of the eight dimensions, i.e. the dimensions of innovation, being active, risking, strategic orientation, the resource-based orientation, management structure, bounces philosophy, and entrepreneurial culture will be expressed. The following table shows the mean and standard deviation of dimensions.

Table 5. The mean and standard deviation of CE dimensions One-Sample Statistics

\begin{tabular}{ccccc}
\hline & $\mathrm{N}$ & Mean & Std. Deviation & Std. Error Mean \\
\hline VAR00001 & 137 & 5.3452 & 1.31629 & .13033 \\
VAR00002 & 137 & 5.1538 & 1.16957 & .11580 \\
VAR00003 & 137 & 3.4167 & 1.49228 & .14776 \\
VAR00004 & 137 & 3.8824 & 1.49993 & .14852 \\
VAR00005 & 137 & 3.7731 & 1.86017 & .18418 \\
VAR00006 & 137 & 4.9477 & 1.57136 & .15559 \\
VAR00007 & 137 & 5.6242 & 1.83471 & .18166 \\
VAR00008 & 137 & 3.6634 & 1.90134 & .08925 \\
\hline
\end{tabular}

In the first part, the results of data analysis using one-way T-test based on the designed assumption will be examined. Because the questionnaire was based on the range of 7 options, based on the statistical books mean should be considered the number 4.

Example Hypothesis: the CE innovation from housing investment employees and managers perspective is in a good condition. To investigate this hypothesis, statistical hypothesis was designed as follows.

The CE innovation from housing investment employees and managers perspective is not in a good condition. The CE innovation from housing investment employees and managers perspective is in a good condition. 
Table 6. T-test for all dimensions of CE inventory

\begin{tabular}{|c|c|c|c|c|c|c|}
\hline \multicolumn{7}{|l|}{ One-Sample Test } \\
\hline \multirow{3}{*}{ Dimension } & \multicolumn{6}{|c|}{ Test Value $=4$} \\
\hline & \multirow[t]{2}{*}{$\mathrm{T}$} & \multirow[t]{2}{*}{ df } & \multirow[t]{2}{*}{ Sig. (2-tailed) } & \multirow[t]{2}{*}{$\begin{array}{c}\text { Mean } \\
\text { Difference }\end{array}$} & \multicolumn{2}{|c|}{$\begin{array}{l}\text { 95\% Confidence Interval of the } \\
\text { Difference }\end{array}$} \\
\hline & & & & & Lower & Upper \\
\hline Innovation & 2.347 & 136 & .020 & -.21158 & .3896 & .0335 \\
\hline Being active & 2.017 & 136 & .045 & -.18669 & .3696 & .0038 \\
\hline Risking & -.141 & 136 & .888 & -.01391 & -.2087 & .1809 \\
\hline Strategic orientation & -.472 & 136 & .638 & -.04257 & -.2209 & .1357 \\
\hline $\begin{array}{l}\text { Resource-based } \\
\text { orientation }\end{array}$ & -1.601 & 136 & .112 & -.16667 & -.3724 & .0391 \\
\hline $\begin{array}{l}\text { Management } \\
\text { Structure }\end{array}$ & 2.248 & 136 & .026 & .23377 & .0284 & .4392 \\
\hline Bounes Philosophy & 1.274 & 136 & .004 & .14935 & .0822 & .3809 \\
\hline $\begin{array}{l}\text { Entrepreneurial } \\
\text { culture }\end{array}$ & -2.218 & 136 & .091 & .13567 & -.0542 & -.5382 \\
\hline
\end{tabular}

In the above table dimensons with a significance level of less than 0.05 are in good condition, with four dimensions in this category. On the other hand, with aglance at the table of the mean and the standard deviation it is evident that the dimensions of innovation, being active, and management structure and bounes philosophy show much higher means than the other dimensions. Now, dimensions that lack good condition should be added to Quality Function Deployment model to develop strategies. If QFD is used in a manufacturing company in the manufacturing sector to improve the quality of a product, two quality stages or two quality boxes should be passed by, but in the non-manufacturing sector or service providing, due to the length of completion of this model using expert opinion, only a quality box can provide researcher with adequate results. It can be noted that the model is used in one stage in many non- manufacturing organizations studies including that of organizational agility (Sazvar, 2010) and (Bottani, 2009) and Jahangardi (Tan \& Pawitra, 2001). Also, due to the length and difficulty of completing the model, the participation of 5 model subjects is adequate and the number of participants should be odd for each indicator, the number of higher frequency is entered in the QFD table (Sazvar, 2010) and (Bottani, 2009), in this study, 11 of senior executives of the two surveyed companies cooperated with the researchers in the completion of this model. 

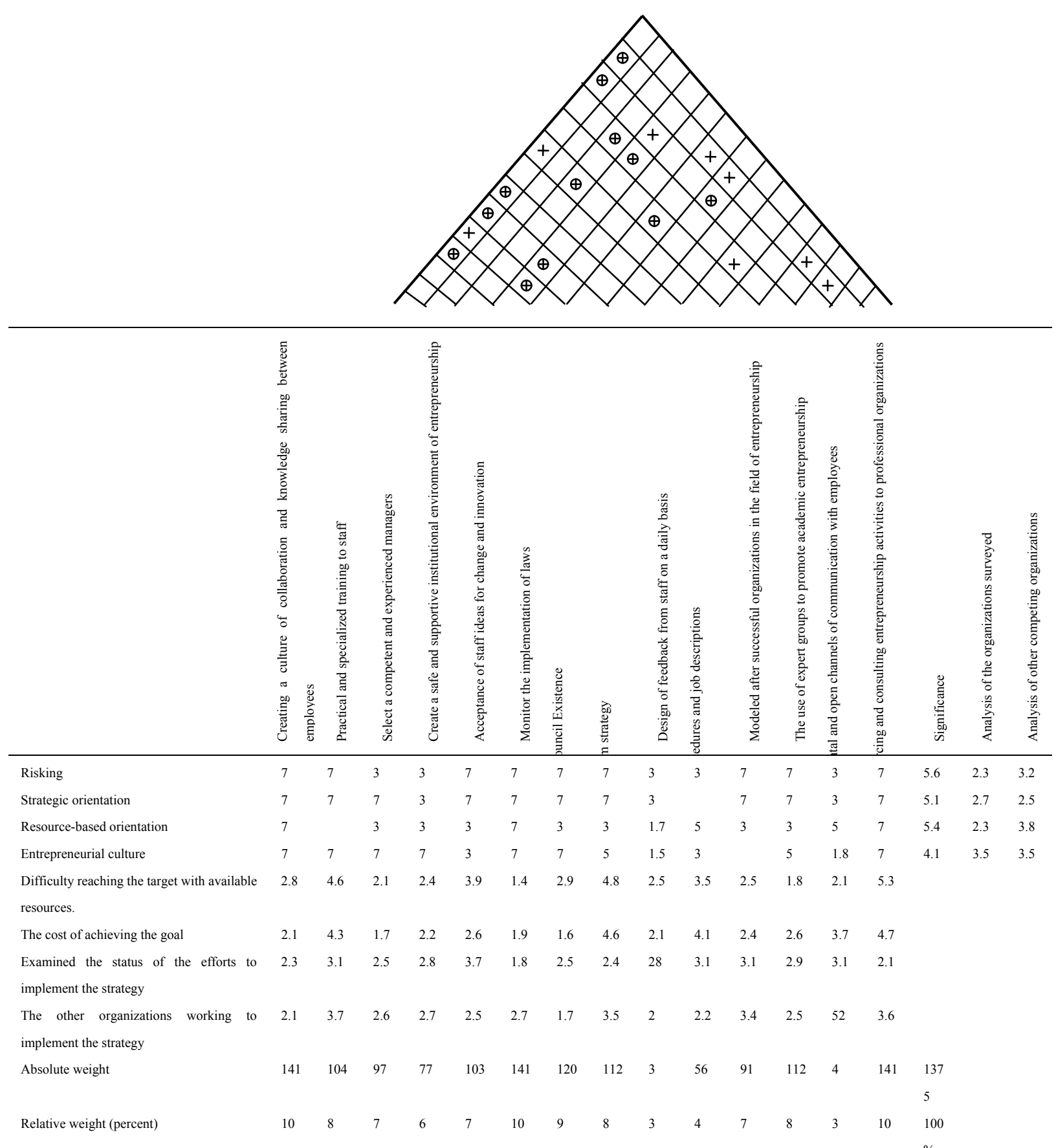

Figure 1. The quality box of CE critical dimension conversion into improvement strategies

As seen in the chart above the quality box inputs determination is prerequisite along the road to recovery, which according to previous stage, four critical dimensions are being considered as input.

The first step is to identify the factors affecting 4 input parameters:

A number of 11 of senior executives of the two surveyed companies cooperated with the researchers and 14 improvement strategies were identified as follows each can be effective in improving the number of input parameters:

1- Create a culture of collaboration and knowledge sharing between employees

2- Practical training and entrepreneurial expertise to staff 
3- Select competent and experienced managers

4- Create a safe and supportive institutional environment of entrepreneurship

5- The acceptance for staff ideas of change and innovation

6- Monitoring the implementation of laws

7- Existence of a CE Policy Council

8- CE long-term strategy making

9- The design of feedback system of staff on a daily basis

10- Existence of defined procedure and job description

11- Modeled after successful organizations in the field of entrepreneurship

12- The use of expert groups to promote academic CE

13- Create open and horizontal channels of communication with employees

14- Outsourcing CE consulting to professional organizations

But the next question is that wither these strategies have a negative effect on each other or not?

The second stage, quality home roof: at this stage we need to look for this purpose that what are 14 strategies effects on each other. If two or more indicators have a negative effect, one should be removed. Using expert opinion, and as specified in the table, there is no negative effects. Note that, high positive impact is shown by the sign (positive circle) and moderate positive impact with sign (positive).

The third step, the significance level: At this stage experts gave each of five indexes a score ranged from 1 (very low) to 7(very high) with mean are shown in Table. Also, the surveyed organizations and other organizations states in each of the parameters were evaluated according to the 11 directors perspectives, according to the same spectrum.

The fourth phase, the effects matrix: the effects of each of the 14 strategies on the 5 indexes should be specified in the Quality middle box matrix. The digit 7 means the most effective 7, 5 the average impact and 3 is weak impact. Also blank box represents the ineffectiveness. As observed, indexes such as to create a culture of participation and knowledge sharing among staff, monitor the implementation of laws and outsourcing CE consulting to professional organizations with large impact on all inputs are of high value.

The fifth stage, weights: In the lower part of the quality box, the difficulty of achieving strategies, their costs and the state of surveyed cooperation and other operations in their previous efforts in achieving these strategies should be identified. These numbers are based on a range of 7 options. The relative weight of each indicator is obtained based on effects and input significance matrix multiplication.

\section{Conclusion}

In the current study, Quality Function Deployment model have been completed through interviews with 11 managers of two surveyed organizations and resulted in significant outcomes. First, all strategies for improvement offered by the group, were put together, and similar indices were modulated and indicators that have been mentioned only once or those were not applied have been removed.

Thus a number of 14 main strategies were introduced. Quality home roofs can provide researcher with important result. So that if one of the strategies is to have a positive impact on a greater number of strategies, it should be considered as a basic solution because its implementation is virtually gets a number of other strategies closer to their targets. As observed, three strategies have the greatest influence:

1- Create a culture of collaboration and knowledge sharing between employees

2- Open and horizontal communication channels with staff

3- Safe and supportive institutional environment of entrepreneurship

It should be noted ranking strategies can be based on many factors such as cost of achieving the goal or difficulty to implement a strategy. Based on the cost of achieving the goal, the priority to implement strategies is as the following:

1- Design of feedback from staff on a daily basis

2- Existence of a CE Policy Council

3- Choosing a competent and experienced managers 
Based on the degree of difficulty reaching the target, indicators as (monitoring to implementation of laws) and (design a feedback system from employees on a daily basis), are the priorities to implement. However, care must be taken that based on specific policies, combining both criteria of cost difficulty can be used or prefer one over the other. On the other hand, the strategies relative and absolute weight are other measures for ranking of the three strategies (monitoring to implementation of laws), (creating a culture of collaboration and knowledge sharing between employees) and (Outsourcing CE consulting to professional organizations) have the highest weight and influence and thus with their implementation will lead to the recovery of more input parameters and $\mathrm{CE}$ will be promoted with a greater intensity.

\section{References}

Beigi, N., Abolreza. Safari, S., \& Mohammadi, M. (2010). The study of the effect of needs kinds on the willingness of staff of CE)", (Case Study: Headquarters NIOC). Journal of Human Resources Management in the Oil Industry, 13, 7-36.

Bottani, E. (2009). A fuzzy QFD approach to achieve agility. International Journal of Production Economics, 119, 380-391.

Farhangi, Ali A., \& Safarzadeh, H. (2005). Design and explain the pattern of corporate communications and corporate entrepreneurship process. Scientific Journal, 14, 1-20.

Farhangi, Ali A., \& Safarzadeh, H. (2007). Entrepreneurship, (concepts, ideas, models and applications), the Institute of Labor and Social Security, Tehran

Fox, Jolie. M. (2008). Organizational entrepreneurship and organizational performance linkage in university extension, USASBE 2008 Proceedings, pp. 429. 459.

Ghahramani, M., Pardakhtchi, Mohammad H., \& Hossein Zade, T. (2010). Organizational culture and its relation to CE. Public management perspective Quarterly, 1, 25-39.

Lumpkin, G. T., \& Lichtenstein, B. B. (2005). The role of organizational learning in the opportunity-recognition process. Entrepreneurship Theory \& Practice, 29(4), 451-472.

Montoro-Sánchez, Á., \& Soriano, Domingo R. (2011). Human resource management and corporate entrepreneurship. International Journal of Manpower, 32(1), 6-13.

Peltola, S. (2012). Can an old firm learn new tricks? A corporate entrepreneurship approach to organizational renewal. Business Horizons, 55, 43-51.

Rahmati Zanjan Talab, Farshad. (2011). Design an indigenous model for promoting CE in cultural institutions and organizations (Case Study: Islamic Azad University, Science and Research Branch, Tehran). Cultural Management Journal, V(XI), 89-108.

Reza Zadeh, H. (2003). The study of relationship between structure and CE (case study of Tehran Social Security Administration), Master Thesis, Tehran University

Sazvar, A. (2010). Improve organizational agility in higher education institutions using quality function deployment. MS Thesis, School of Management, Quash PNU.

Tan, K. C., \& Pawitra, T. A. (2001). Integrating SERVQUAL and Kano's model into QFD for service excellence development. Managing Service Quality, 11(6), 418-430.

\section{Copyrights}

Copyright for this article is retained by the author(s), with first publication rights granted to the journal.

This is an open-access article distributed under the terms and conditions of the Creative Commons Attribution license (http://creativecommons.org/licenses/by/3.0/). 